\title{
A new combinatorial identity for unicellular maps, via a direct bijective approach.
}

\author{
Guillaume Chapuy ${ }^{1}$ \\ ${ }^{1}$ Laboratoire d'Informatique de l'École Polytechnique, 91128 Palaiseau Cedex, France.
}

\begin{abstract}
We give a bijective operation that relates unicellular maps of given genus to unicellular maps of lower genus, with distinguished vertices. This gives a new combinatorial identity relating the number $\epsilon_{g}(n)$ of unicellular maps of size $n$ and genus $g$ to the numbers $\epsilon_{j}(n)$ 's, for $j<g$. In particular for each $g$ this enables to compute the closed-form formula for $\epsilon_{g}(n)$ much more easily than with other known identities, like the Harer-Zagier formula. From the combinatorial point of view, we give an explanation to the fact that $\epsilon_{g}(n)=R_{g}(n) \operatorname{Cat}(n)$, where Cat $(n)$ is the $n$-th Catalan number and $R_{g}$ is a polynomial of degree $3 g$, with explicit interpretation.

Résumé. On décrit une opération bijective qui relie les cartes à une face de genre donné à des cartes à une face de genre inférieur, portant des sommets marqués. Cela conduit à une nouvelle identité combinatoire reliant le nombre $\epsilon_{g}(n)$ de cartes à une face de taille $n$ et genre $g$ aux nombres $\epsilon_{j}(n)$, pour $j<g$. En particulier, pour tout $g$, cela permet de calculer la formule close donnant $\epsilon_{g}(n)$ bien plus facilement qu'à l'aide des autres identités connues, comme la formule d'Harer-Zagier. Du point de vue combinatoire, nous donnons une explication au fait que $\epsilon_{g}(n)=$ $R_{g}(n)$ Cat $(n)$, où $\operatorname{Cat}(n)$ est le nième nombre de Catalan et $R_{g}$ est un polynôme de degré $3 g$, à l'interprétation explicite.
\end{abstract}

Keywords: Polygon gluings, combinatorial identity, bijection.

\section{Introduction.}

A unicellular map is a graph embedded on a compact orientable surface, in such a way that its complement is a topological polygon. Equivalently, a unicellular map can be viewed as a polygon, with an even number of edges, in which edges have been pasted pairwise in order to create a closed orientable surface. The number of handles of this surface is called the genus of the map.

These objects are reminiscent in combinatorics, and have been considered by several authors, with different methods, and under different names. According to the context, unicellular maps can also be called polygon gluings, one-border ribbon graphs, or factorisations of a cycle. The most famous example of unicellular maps are planar unicellular maps, which, from Jordan's lemma, are exactly plane trees, enumerated by the Catalan numbers.

The first result in the enumeration of unicellular maps in positive genus was obtained by Lehman and Walsh [WL72]. Using a direct recursive method, relying on multivariate recurrence equations, they expressed the number $\epsilon_{g}(n)$ of unicellular maps with $n$ edges on a surface of genus $g$ as follows:

$$
\epsilon_{g}(n)=\sum_{\gamma \vdash g} \frac{(n+1) \ldots(n+2-2 g-l(\gamma))}{2^{2 g} \prod_{i} c_{i} !(2 i+1)^{c_{i}}} \operatorname{Cat}(n),
$$

1365-8050 @ 2009 Discrete Mathematics and Theoretical Computer Science (DMTCS), Nancy, France 
where the sum is taken over partitions $\gamma$ of $g, c_{i}$ is the number of parts $i$ in $\gamma, l(\gamma)$ is the total number of parts, and Cat $(n)$ is the $n$-th Catalan number. This formula has been extended by other authors ([GS98]).

Later, Harer and Zagier [HZ86], via matrix integrals techniques, obtained the two following equations, known respectively as the Harer-Zagier recurrence and the Harer-Zagier formula:

$$
\begin{array}{r}
(n+1) \epsilon_{g}(n)=2(2 n-1) \epsilon_{g}(n-1)+(2 n-1)(n-1)(2 n-3) \epsilon_{g-1}(n-2), \\
\sum_{g \geq 0} \epsilon_{g}(n) y^{n+1-2 g}=\frac{(2 n) !}{2^{n} n !} \sum_{i \geq 1} 2^{i-1}\left(\begin{array}{c}
n \\
i-1
\end{array}\right)\left(\begin{array}{c}
y \\
i
\end{array}\right) .
\end{array}
$$

Formula 3 has been retrieved by several authors, by various techniques. A combinatorial interpretation of this formula was given by Lass [Las01], and the first bijective proof was given by Goulden and Nica [GN05]. Generalizations were given for bicolored, or multicolored maps [Jac87, SV08].

The purpose of this paper is to give a new angle of attack to the enumeration of unicellular maps, at a level which is much more combinatorial than what existed before. Indeed, until now no bijective proof (or combinatorial interpretation) of Formulas 1 and 2 are known. As for Formula 3 , it is concerned with some generating polynomial of the numbers $\epsilon_{g}(n)$ : in combinatorial terms, the bijections in [GN05, SV08] concern maps which are weighted according to their genus, by an additional coloring of their vertices, but the genus does not appear explicitely in the constructions. Moreover, these bijections concern those weighted maps, more than the unicellular maps themselves.

On the contrary, this article is concerned with the structure of unicellular maps themselves, at given genus. We investigate in details the way the unique face of such a map "interwines" with itself in order to create the handles of the surface. We show that, in each unicellular map of genus $g$, there are $2 g$ "special places", which we call trisections, that concentrate, in some sense, the handles of the surface. Each of these places can be used to slice the map to a unicellular map of lower genus. Conversely, we show that a unicellular map of genus $g$ can always be obtained in $2 g$ different ways by gluing vertices together in a map of lower genus. In terms of formulas, this leads us to the new combinatorial identity:

$$
\begin{aligned}
2 g \cdot \epsilon_{g}(n) & =\left(\begin{array}{c}
n+3-2 g \\
3
\end{array}\right) \epsilon_{g-1}(n)+\left(\begin{array}{c}
n+5-2 g \\
5
\end{array}\right) \epsilon_{g-2}(n)+\ldots+\left(\begin{array}{c}
n+1 \\
2 g+1
\end{array}\right) \epsilon_{0}(n) \\
& =\sum_{p=0}^{g-1}\left(\begin{array}{c}
n+1-2 p \\
2 g-2 p+1
\end{array}\right) \epsilon_{p}(n) .
\end{aligned}
$$

This identity enables to compute, for each $g$, the closed formula giving $\epsilon_{g}(n)$ in terms of the $n$-th Catalan number much more easily than Formulas $1 / 233$ (indeed, even Formula 1 has quite a big number of terms). In combinatorial terms, this enables to perform either exhaustive or random sampling of unicellular maps of given genus and size easily. When iterated, our bijection really shows that all unicellular maps can be obtained in a canonical way from plane trees by successive gluings of vertices, hence giving the first explanation to the fact that $\epsilon_{g}(n)$ is the product of a polynomial in $n$ by the $n$-th Catalan number.

Acknowledgements. I am indebted to Olivier Bernardi, and to my advisor Gilles Schaeffer, for very stimulating discussions. 




$$
\mid \begin{aligned}
& H=\llbracket 1,22 \rrbracket \\
& \alpha=(1,22)(2,5)(3,11)(4,12)(6,21)(7,16) \\
& (8,9)(10,15)(13,18)(14,19)(17,20) \\
& \sigma=(1,5,21)(2,11,4)(3,12,18,14,10) \\
& (6,16,20)(7,9,15)(8)(13,19,17)(22) \\
& \gamma=\alpha \sigma=(1,2,3, \ldots, 22)
\end{aligned}
$$

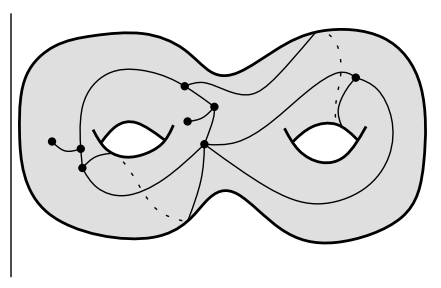

Figure 1: A unicellular map with 11 edges, 8 vertices, and genus 2: (a) ribbon graph; (b) permutations; (c) topological embedding.

\section{Unicellular maps.}

\subsection{Permutations and ribbon graphs.}

Rather than talking about topological embeddings of graphs, we work with a combinatorial definition of unicellular maps:

Definition 1. A unicellular map $M$ of size $n$ is a triple $M=(H, \alpha, \sigma)$, where $H$ is a set of cardinality $2 n, \alpha$ is an involution of $H$ without fixed points, and $\sigma$ is a permutation of $H$ such that $\gamma=\alpha \sigma$ has only one cycle. The elements of $H$ are called the half-edges of $M$. The cycles of $\alpha$ and $\sigma$ are called the edges and the vertices of $M$, respectively, and the permutation $\gamma$ is called the face of $M$.

Given a unicellular map $M=(H, \sigma, \alpha)$, its associated (multi)graph $G$ is the graph whose edges are given by the cycles of $\alpha$, vertices by the cycles of $\sigma$, and the natural incidence relation $v \sim e$ if $v$ and $e$ share an element. Moreover, we draw each edge of $G$ as a ribbon, where each side of the ribbon represents one half-edge; we decide which half-edge corresponds to which side of the ribbon by the convention that, if a half-edge $h$ belongs to a cycle $e$ of $\alpha$ and $v$ of $\sigma$, then $h$ is the right-hand side of the ribbon corresponding to $e$, when considered entering $v$. Furthermore, we draw the graph $G$ in such a way that around each vertex $v$, the counterclockwise ordering of the half-edges belonging to the cycle $v$ is given by that cycle: we obtain a graphical object called the ribbon graph associated to $M$, as in Figure 11a). Observe that the unique cycle of the permutation $\gamma=\alpha \sigma$ interprets as the sequence of half-edges visited when making the tour of the graph, keeping the graph on its left.

A rooted unicellular map is a unicellular map carrying a distinguished half-edge $r$, called the root. These maps are considered up to relabellings of $H$ preserving the root, i.e. two rooted unicellular maps $M$ and $M^{\prime}$ are considered the same if there exists a permutation $\pi: H \rightarrow H^{\prime}$, such that $\pi(r)=r^{\prime}$, $\alpha=\pi^{-1} \alpha^{\prime} \pi$, and $\sigma=\pi^{-1} \sigma^{\prime} \pi$. In this paper, all unicellular maps will be rooted, even if not stated.

Given a unicellular map $M$ of root $r$ and face $\gamma=\alpha \sigma$, we define the linear order $<_{M}$ on $H$ by setting: $r<_{M} \gamma(r)<_{M} \gamma_{2}(r)<_{M} \ldots<_{M} \gamma^{2 n-1}(r)$.

In other words, if we relabel the half-edge set $H$ by elements of $\llbracket 1,2 n \rrbracket$ in such a way that the root is 1 and the tour of the face is given by the permutation $(1, \ldots, 2 n)$, the order $<_{M}$ is the natural order on the integers. However, since in this article we are going to consider maps with a fixed half-edge set, but a changing permutation $\gamma$, it is more convenient (and prudent) to define the order $<_{M}$ in this way.

Unicellular maps can also be interpreted as graphs embedded in a topological surface, in such a way that the complement of the graph is a topological polygon. If considered up to homeomorphism, and suitably rooted, these objects are in bijection with ribbon graphs. See [MT01], or the example of Figure 11.c). The 
genus of a unicellular map is the genus, or number of handles, of the corresponding surface. If a unicellular map of genus $g$ has $n$ edges and $v$ vertices, then Euler's characteristic formula says that $v=n+1-2 g$. From a combinatorial point of view, this last equation can also be taken as a definition of the genus.

\subsection{The gluing operation.}

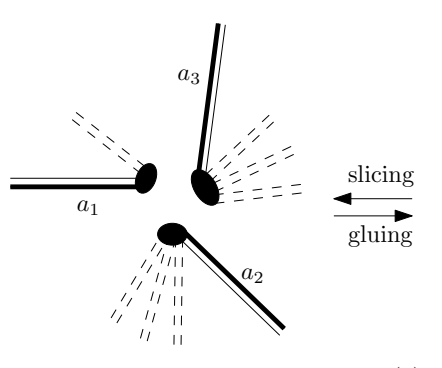

(a)

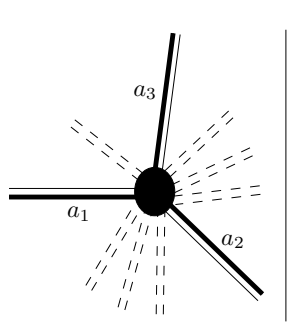

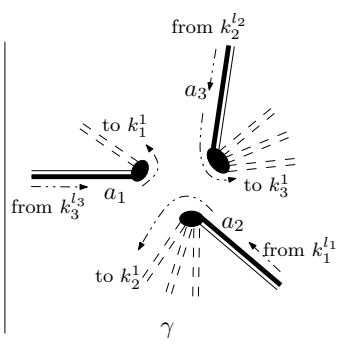

(b)

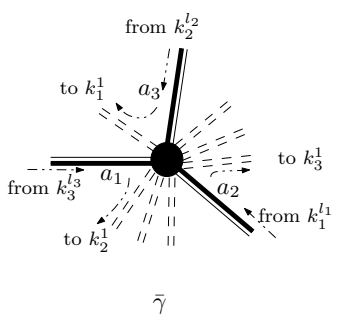

Figure 2: (a) The gluing and slicing operations. (b) The "proof" of Lemma 1

We let $M=(H, \alpha, \sigma)$ be a unicellular map of genus $g$, and $a_{1}<_{M} a_{2}<_{M} a_{3}$ be three half-edges of $M$ belonging to three distinct vertices. Each half-edge $a_{i}$ belongs to some vertex $v_{i}=\left(a_{i}, h_{i}^{1}, \ldots h_{i}^{m_{i}}\right)$, for some $m_{i} \geq 0$. We define the permutation

$$
\bar{v}:=\left(a_{1}, h_{2}^{1}, \ldots h_{2}^{m_{2}}, a_{2}, h_{3}^{1}, \ldots h_{3}^{m_{3}}, a_{3}, h_{1}^{1}, \ldots h_{1}^{m_{1}}\right),
$$

and we let $\bar{\sigma}$ be the permutation of $H$ obtained by deleting the cycles $v_{1}, v_{2}$, and $v_{3}$, and replacing them by $\bar{v}$. The transformation mapping $\sigma$ on $\bar{\sigma}$ interprets combinatorially as the gluing of the three half-edges $a_{1}, a_{2}, a_{3}$, as shown on Figure 2(a). We have:

Lemma 1. The map $\bar{M}:=(H, \alpha, \bar{\sigma})$ is a unicellular map of genus $g+1$. If we let $\gamma=\alpha \sigma=$ $\left(a_{1}, k_{1}^{1}, \ldots k_{1}^{l_{1}}, a_{2}, k_{2}^{1}, \ldots k_{2}^{l_{2}}, a_{3}, k_{3}^{1}, \ldots k_{3}^{l_{3}}\right)$ be the face permutation of $M$, then the face of $\bar{M}$ is given by:

$$
\bar{\gamma}=\left(a_{1}, k_{2}^{1}, \ldots k_{2}^{l_{2}}, a_{3}, k_{1}^{1}, \ldots k_{1}^{l_{1}}, a_{2}, k_{3}^{1}, \ldots k_{3}^{l_{3}}\right)
$$

Proof: In order to prove that $M$ is a well-defined unicellular map, it suffices to check that its face is given by the long cycle $\bar{\gamma}$ given in the lemma. This is very easy to check by observing that the only half-edges whose image is not the same by $\gamma$ and by $\bar{\gamma}$ are the three half-edges $a_{1}, a_{2}, a_{3}$, and that by construction $\bar{\gamma}\left(a_{i}\right)=\alpha \bar{\sigma}\left(a_{i}\right)=\alpha \sigma\left(a_{i+1}\right)=\gamma\left(a_{i+1}\right)$. For a more "visual" explanation, see Figure 2 b).

Now, by construction, $M^{\prime}$ has two less vertices than $M$, and the same number of edges, so from Euler's formula it has genus $g+1$ (intuitively, the gluing operation has created a new "handle").

\subsection{Some intertwining hidden there, and the slicing operation.}

The aim of this paper is to show that all unicellular maps of genus $g+1$ can be obtained in some canonical way from unicellular maps of genus $g$ from the operation above. This needs to be able to "revert" (in some 
(a)

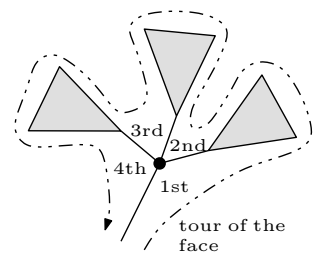

(b)

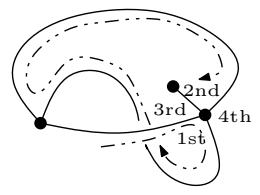

Figure 3: (a) In a plane tree, the tour of the face always visits the half-edges around one vertex in counterclockwise order; (b) in positive genus (here in genus 1), things can be different.

sense) the gluing operation, hence to be able to determine, given a map of genus $g+1$, which vertices may be "good candidates" to be sliced-back to a map of lower genus.

Observe that in the unicellular map $\bar{M}$ obtained after the gluing operation, the three half-edges $a_{1}$, $a_{2}, a_{3}$ appear in that order around the vertex $\bar{v}$, whereas they appear in the inverse order in the face $\bar{\gamma}$. Observe also that this is very different from what we observe in the planar case: if one makes the tour of a plane tree, with the tree on its left, then one necessarily visits the different half-edges around each vertex in counterclockwise order (see Figure 3). Informally, one could hope that, in a map of positive genus, those places where the vertex-order does not coincide with the face-order hide some "intertwining" (some handle) of the map, and that they may be used to slice-back the map to lower genus.

We now describe the slicing operation, which is nothing but the gluing operation, taken at reverse. We let $\bar{M}=(H, \alpha, \bar{\sigma})$ be a map of genus $g+1$, and three half-edges $a_{1}, a_{2}, a_{3}$ belonging to a same vertex $\bar{v}$ of $\bar{M}$. We say that $a_{1}, a_{2}, a_{3}$ are intertwined if they do not appear in the same order in $\bar{\gamma}=\alpha \bar{\sigma}$ and in $\bar{\sigma}$. In this case, we write $\bar{v}=\left(a_{1}, h_{2}^{1}, \ldots h_{2}^{m_{2}}, a_{2}, h_{3}^{1}, \ldots h_{3}^{m_{3}}, a_{3}, h_{1}^{1}, \ldots h_{1}^{m_{1}}\right)$, and we let $\sigma$ be the permutation of $H$ obtained from $\bar{\sigma}$ by replacing the cycle $\bar{v}$ by the product $\left(a_{1}, h_{1}^{1}, \ldots h_{m_{1}}^{1}\right)\left(a_{2}, h_{1}^{2}, \ldots h_{m_{2}}^{2}\right)\left(a_{3}, h_{1}^{3}, \ldots h_{m_{3}}^{3}\right)$.

Lemma 2. The map $M=(H, \alpha, \sigma)$ is a well-defined unicellular map of genus $g$. If we let $\bar{\gamma}=$ $\left(a_{1}, k_{2}^{1}, \ldots k_{2}^{l_{2}}, a_{3}, k_{1}^{1}, \ldots k_{1}^{l_{1}}, a_{2}, k_{3}^{1}, \ldots k_{3}^{l_{3}}\right)$ be the unique face of $\bar{M}$, then the unique face of $M$ is given by: $\gamma=\alpha \sigma=\left(a_{1}, k_{1}^{1}, \ldots k_{1}^{l_{1}}, a_{2}, k_{2}^{1}, \ldots k_{2}^{l_{2}}, a_{3}, k_{3}^{1}, \ldots k_{3}^{l_{3}}\right)$.

The gluing and slicing operations are inverse one to the other.

Proof: The proof is the same as in Lemma11 it is sufficient to check the expression given for $\gamma$ in terms of $\bar{\gamma}$, which is easily done by checking the images of $a_{1}, a_{2}, a_{3}$.

\subsection{Around one vertex: up-steps, down-steps, and trisections.}

Let $M=(H, \alpha, \sigma)$ be a map of face permutation $\gamma=\alpha \sigma$. For each vertex $v$ of $M$, we let $\min _{M}(v)$ be the minimal half-edge belonging to $v$, for the order $<_{M}$. Equivalently, $\min _{M}(v)$ is the first half-edge from which one reaches $v$ when making the tour of the map, starting from the root. Given a half-edge $h \in H$, we note $V(h)$ the unique vertex it belongs to (i.e. the cycle of $\sigma$ containing it).

Definition 2. We say that a half-edge $h \in H$ is an up-step if $h<_{M} \sigma(h)$, and that it is a down-step if $\sigma(h) \leq_{M} h$. A down-step $h$ is called a trisection if $\sigma(h) \neq \min _{M} V(h)$, i.e. if $\sigma(h)$ is not the minimum half-edge inside its vertex.

As illustrated on Figure 3, trisections are specific to the non-planar case (there are no trisections in a plane tree), and one could hope that trisections "hide" (in some sense) the handles of the surface. Before making this more precise, we state the following lemma, which is the cornerstone of this paper: 


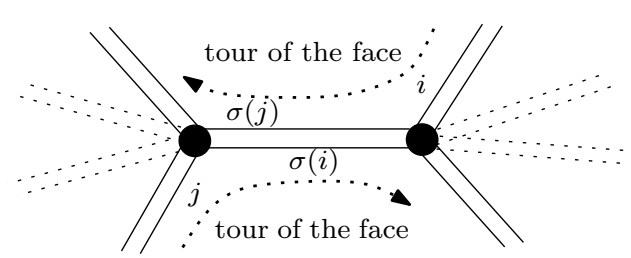

Figure 4: The main argument in the proof of the trisection lemma: the tour of the face visits $i$ before $\sigma(i)$ if and only if it visits $\sigma(j)$ before $j$, unless $\sigma(i)$ or $\sigma(j)$ is the root of the map.
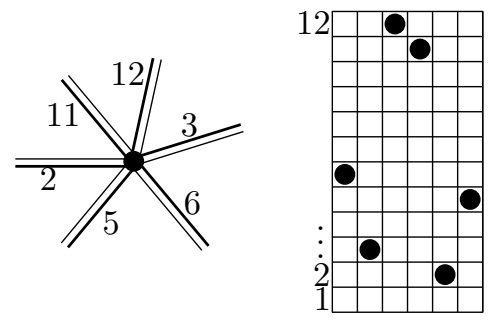

Figure 5: A vertex $(6,3,12,11,2,5)$ in a map with 12-half-edges, and its diagram representation (the marked half-edge is 6).

Lemma 3 (The trisection lemma). Let $M$ be a unicellular map of genus $g$. Then $M$ has exactly $2 g$ trisections.

Proof: We let $M=(H, \alpha, \sigma)$, and $\gamma=\alpha \sigma$. We let $n_{+}$and $n_{-}$denote the number of up-steps and downsteps, respectively. Then, we have $n_{-}+n_{+}=2 n$, where $n$ is the number of edges of $M$. Now, let $i$ be a half-edge of $M$, and $j=\sigma^{-1} \alpha \sigma(i)$. Observe that we have $\sigma(j)=\gamma(i)$, and $\gamma(j)=\sigma(i)$. Graphically, $i$ and $j$ lie in two "opposite" corners of the same edge, as shown on Figure 4 On the picture, it seems clear that if we visit $i$ before $\sigma(i)$, then we necessarily visit $\sigma(j)$ before $j$ (except if the root is one of these four half-edges) so that, roughly, there must be almost the same number of up-steps and down-steps. More precisely, let us distinguish three cases.

First, assume that $i$ is an up-step. Then we have $i<_{M} \sigma(i)=\gamma(j)$. Now, by definition of the total order $<_{M}, i<_{M} \gamma(j)$ implies that $\gamma(i) \leq_{M} \gamma(j)$. Hence, $\sigma(j) \leq_{M} \gamma(j)$, which, by definition of $<_{M}$ again, implies that $\sigma(j) \leq_{M} j$ (here, we have used that $\sigma(j) \neq \gamma(j)$ since $\alpha$ has no fixed point). Hence, if $i$ is an up-step, then $j$ is a down-step.

Second, assume that $i$ is a down-step, and that $\gamma(j)$ is not equal to the root of $M$. In this case, we have $j<_{M} \gamma(j)$, and $\gamma(j)=\sigma(i) \leq_{M} i=\sigma(j)$. Hence $j<_{M} \sigma(j)$, and $j$ is an up-step.

The third and last case is when $i$ is a down step, and $\gamma(j)$ is the root $r$ of $M$. In this case, $j$ is the maximum element of $H$ for the order $<_{M}$, so that it is necessarily a down-step.

Therefore we have proved that each edge of $M$ (more precisely, each cycle of $\sigma^{-1} \alpha \sigma$ ) is associated to one up-step and one down-step, except a special one that has two down-steps. Consequently, there are exactly two more down-steps that up-steps in the map $M$, i.e.: $n_{-}=n_{+}+2$. Recalling that $n_{-}+n_{+}=2 n$, this gives $n_{-}=n+1$.

Finally, each vertex of $M$ carries exactly one down-step which is not a trisection (its minimal halfedge). Hence, the total number of trisections equals $n_{-}-v$, where $v$ is the number of vertices of $M$. Since from Euler's characteristic formula, $v$ equals $n+1-2 g$, the lemma is proved.

\section{Making the gluing operation injective.}

We have defined above an operation that glues a triple of half-edges, and increases the genus of a map. In this section, we explain that, if we restrict to certain types of triples of half-edges, this operation can be made reversible. 


\subsection{A diagram representation of vertices.}

We first describe a graphical visualisation which should make the exposition more easy. Let $v$ be a vertex of $M$, with a distinguished half-edge $h$. We write $v=\left(u_{0}, u_{1}, \ldots, u_{m}\right)$, with $u_{0}=h$. We now consider a grid with $m+1$ columns and $2 n$ rows. Each row represents an element of $H$, and the rows are ordered from the bottom to the top by the total order $<_{M}$ (for example the lowest row represents the root). Now, for each $i$, inside the $i$-th column, we plot a point at the height corresponding to the half-edge $u_{i}$. We say that the obtained diagram is the diagram representation of $v$, starting from $h$. In other words, if we identify $\llbracket 1,2 n \rrbracket$ with $H$ via the order $<_{M}$, the diagram representation of $v$ is the graphical representation of the sequence of labels appearing around the vertex $v$. If one changes the distinguished half-edge $h$, the diagram representation of $v$ is changed by a circular permutation of its columns. Figure 5 gives an example of such a diagram (where the permutation $\gamma$ is in the form $\gamma=(1,2,3, \ldots)$ ).

The gluing operation is easily visualised on diagrams. We let as before $a_{1}<_{M} a_{2}<_{M} a_{3}$ be three halfedges belonging to distinct vertices in a unicellular map $M$, and we let $\Delta_{1}, \Delta_{2}, \Delta_{3}$ be their corresponding diagrams. We now consider the three horizontal rows corresponding to $a_{1}, a_{2}$, and $a_{3}$ : they separate each diagram $\Delta_{i}$ into four blocks (some of which may be empty). We give a name to each of these blocks: $A_{i}, B_{i}, C_{i}, D_{i}$, from bottom to top, as on Figure 6 (a).

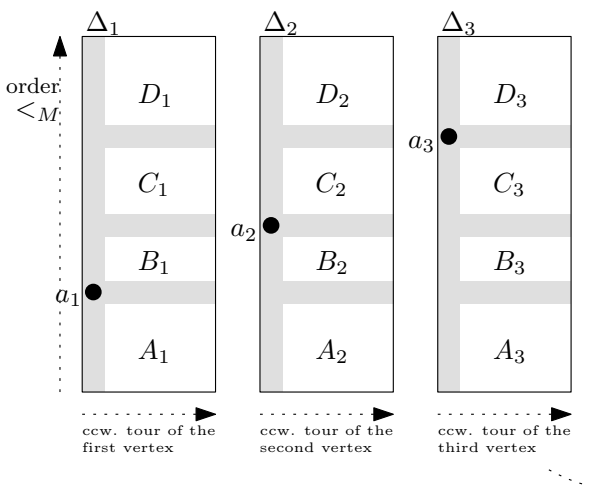

(a)

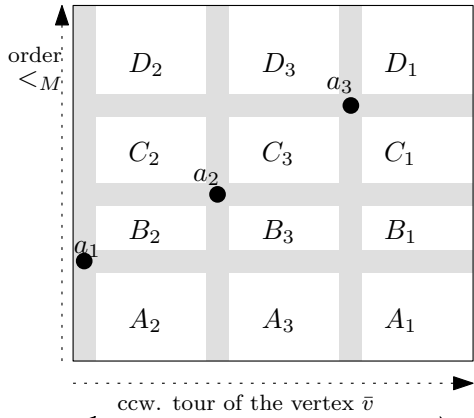

(b)

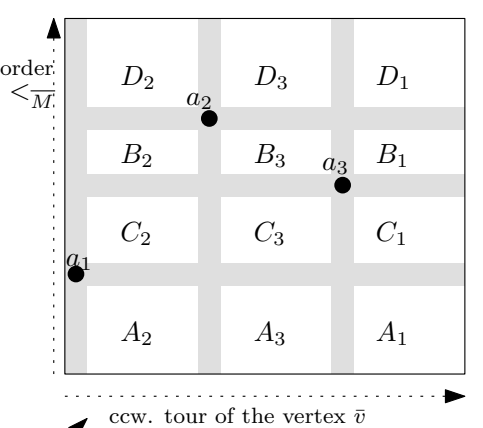

swap the blocks $B$ and $C$, and the rows $a_{2}$ and $a_{3}$ (c)

Figure 6: The gluing operation visualized on diagrams. (a) the diagrams before gluing; (b) a temporary diagram, where we the columns represent the counterclockwise turn around $\bar{v}$, but the rows still represent the original permutation $\gamma$; (c) the final diagram of the new vertex in the new map, where the rows represent the permutation $\bar{\gamma}$.

We now juxtapose $\Delta_{2}, \Delta_{3}, \Delta_{1}$ together, from left to right, and we rearrange the three columns containing $a_{1}, a_{2}, a_{3}$ so that these half-edges appear in that order: we obtain a new diagram (Figure 6 b)), whose columns represent the order of the half-edges around the vertex $\bar{v}$. But the rows of that diagram are still ordered according to the order $<_{M}$. In order to obtain the diagram representing $\bar{v}$ in the new map $\bar{M}$, we have to rearrange the rows according to $<_{\bar{M}}$. We let $A$ be the union of the three blocks $A_{i}$ (and similarly, we define $B, C$, and $D$ ). We know that the face permutation of $M$ has the form $\gamma=\left(-A-, a_{1},-B-, a_{2},-C-, a_{3},-D-\right)$, where by $-A-$, we mean "all the elements of $A$, appearing in a certain order". Now, from the expression of $\bar{\gamma}$ given in Lemma 1 , the permutation $\bar{\gamma}$ is: $\bar{\gamma}=\left(-A-, a_{1},-C-, a_{3},-B-, a_{2},-D-\right)$, where inside each block, the half-edges 
appear in the same order as in $\gamma$. In terms of diagrams, this means that the diagram representing $\bar{v}$ in the new map $\bar{M}$ can be obtained by swapping the block $B$ with the block $C$, and the row corresponding to $a_{2}$ with the one corresponding to $a_{3}$ : see Figure 6(c). To sum up, we have:

Lemma 4. The diagram of the vertex $\bar{v}$ in the map $\bar{M}$ is obtained from the three diagrams $\Delta_{1}, \Delta_{2}, \Delta_{3}$ by the following operations, as represented on Figure 6 .

- Juxtapose $\Delta_{2}, \Delta_{3}, \Delta_{1}$ (in that order), and rearrange the columns containing $a_{1}, a_{2}, a_{3}$, so that they appear in that order from left to right.

- Exchange the blocks $B$ and $C$, and swap the rows containing $a_{2}$ and $a_{3}$.

Observe that, when taken at reverse, Figure 6 gives the way to obtain the diagrams of the three vertices resulting from the slicing operation of three intertwined half-edges $a_{1}, a_{2}, a_{3}$ in the map $\bar{M}$.

\subsection{Gluing three vertices: trisections of type $\mathbf{I}$.}

In this section, we let $v_{1}, v_{2}, v_{3}$ be three distinct vertices in the map $M$. We let $a_{i}:=\min _{M} v_{i}$, and, up to re-arranging the three vertices, we may assume (and we do) that $a_{1}<_{M} a_{2}<_{M} a_{3}$. We let $\Delta_{1}, \Delta_{2}, \Delta_{3}$ be the three corresponding diagrams. Since in each diagram the marked edge is the minimum in its vertex, observe that the blocks $A_{1}, A_{2}, B_{2}, A_{3}, B_{3}, C_{3}$ do not contain any point. We say that they are empty, and we note: $A_{1}=A_{2}=B_{2}=A_{3}=B_{3}=C_{3}=\varnothing$.

We now glue the three half-edges $a_{1}, a_{2}, a_{3}$ in $M$ : we obtain a new unicellular map $\bar{M}$, with a new vertex $\bar{v}$ resulting from the gluing. Now, let $\tau$ be the element preceding $a_{3}$ around $\bar{v}$ in the map $\bar{M}$. Since $A_{3}=B_{3}=C_{3}=\varnothing$, we have either $\tau \in D_{3}$ or $\tau=a_{2}$, so that in both case $a_{3}<_{\bar{M}} \tau$. Moreover, $a_{3}$ in not the minimum inside its vertex (the minimum is $a_{1}$ ). Hence, $\tau$ is a trisection of the map $\bar{M}$. We let $\Phi\left(M, v_{1}, v_{2}, v_{3}\right)=(\bar{M}, \tau)$ be the pair formed by the new map $\bar{M}$ and the newly created trisection $\tau$.

It is clear that given $(\bar{M}, \tau)$, we can inverse the gluing operation. Indeed, it is easy to recover the three half-edges $a_{1}$ (the minimum of the vertex), $a_{3}$ (the one that follows $\tau$ ), and $a_{2}$ (observe that, since $B_{2}$ and $B_{3}$ are empty, $a_{2}$ is the smallest half-edge on the left of $a_{3}$ which is greater than $\left.a_{3}\right)$. Once $a_{1}, a_{2}, a_{3}$ are recovered, it is easy to recover the map $M$ by slicing $\bar{v}$ at those three half-edges. This gives:

Lemma 5. The mapping $\Phi$, defined on the set of unicellular maps with three distinguished (unordered) vertices, is injective.

It is natural to ask for the image of $\Phi$ : in particular, can we obtain all pairs $(\bar{M}, \tau)$ in this way? The answer needs the following definition (see Figure 7):

Definition 3. Let $\bar{M}=(H, \alpha, \bar{\sigma})$ be a map of genus $g+1$, and $\tau$ be a trisection of $\bar{M}$. We let $\bar{v}=V(\tau)$, $b_{1}=\min _{\bar{M}}(\bar{v})$, and we let $\Delta$ be the diagram representation of $\bar{v}$, starting from the half-edge $b_{1}$. We let $b_{3}=\sigma(\tau)$ be the half-edge following $\tau$ around $\bar{v}$, and we let $b_{2}$ be the minimum half-edge among those which appear before $b_{3}$ around $\bar{v}$ and which are greater than $b_{3}$ for the order $<_{\bar{M}}$.

The rows and columns containing $b_{1}, b_{2}, b_{3}$ split the diagram $\Delta$ into twelve blocks, five of which are necessarily empty, as in Figure 7 . We let $K$ be second-from-left and second-from-bottom block. We say that $\tau$ is a trisection of type $\mathbf{I}$ is $K$ is empty, and that $\tau$ is a trisection of type $\mathbf{I I}$ otherwise.

The following proposition is the half way to our main result:

Proposition 1. The mapping $\Phi$ is a bijection between the $\operatorname{set}_{\mathcal{g}}^{3}(n)$ of unicellular maps of genus $g$, with $n$ edges, and three distinguished vertices, and the set $\mathcal{D}_{g+1}^{I}(n)$ of unicellular maps of genus $g+1$ with $n$ edges and a distinguished trisection of type $\mathbf{I}$. 


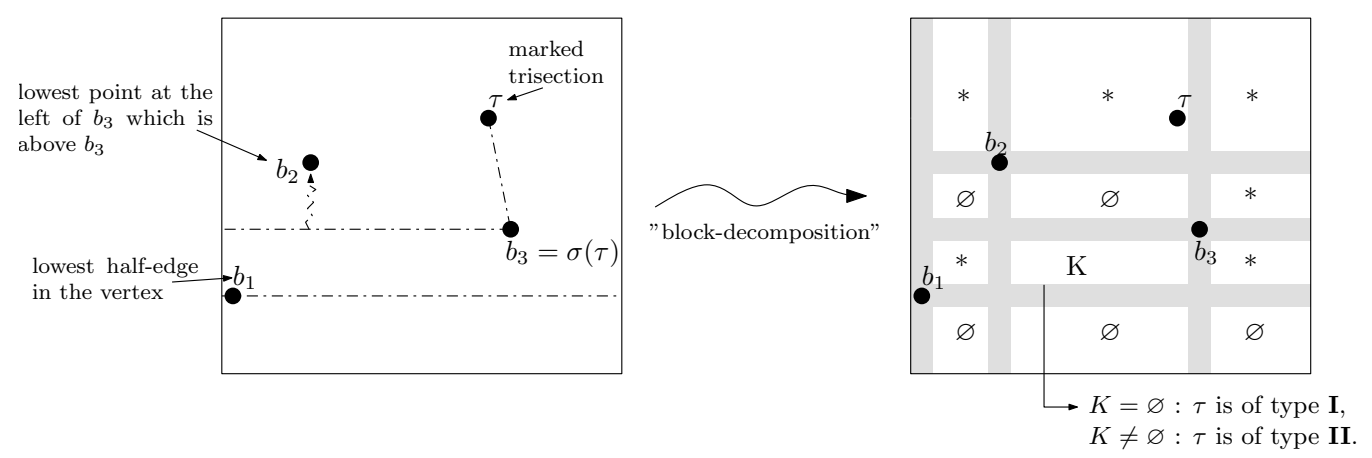

Figure 7: Trisections of type I and II.

Proof: We already know that $\Phi$ is injective.

We let $M$ be a unicellular map of genus $g$ with three distinguished vertices $v_{1}, v_{2}, v_{3}$, and $\bar{M}$ be the map obtained, as above, by the gluing of $M$ by the half-edges $a_{1}=\min _{M} v_{1}, a_{2}=\min _{M} v_{2}, a_{3}=\min _{M} v_{3}$ (we assume again that $a_{1}<_{M} a_{2}<_{M} a_{3}$ ). We let $\bar{\Delta}$ be the diagram representation of the new vertex $\bar{v}$ obtained from the gluing in the map $\bar{M}$, and we use the same notations for the blocks as in Section 3.1 . We also let $\tau=\sigma^{-1}\left(a_{3}\right)$ be the created trisection, and we use the notations of Definition 3 with respect to the trisection $\tau$, so that $b_{3}=a_{3}$. Then, since $a_{1}=\min _{\bar{M}} \bar{v}$, we have $a_{1}=b_{1}$, and since the blocks $B_{2}, B_{3}$, are empty, we have $b_{2}=a_{2}$. Hence, the block $C_{3}$ of Figure 6 (c) coincides with the block $K$ of Figure 7. Since $C_{3}$ is empty, $\tau$ is a trisection of type $\mathbf{I}$. Therefore the image of $\Phi$ is included in $\mathcal{D}_{g+1}^{I}(n)$.

Conversely, let $\bar{M}=(H, \alpha, \bar{\sigma})$ be a map of genus $g+1$, and $\tau$ be a trisection of type $\mathbf{I}$ in $\bar{M}$. We let $b_{1}, b_{2}, b_{3}$ and $K$ be as in Definition 3 First, since $b_{1}<_{\bar{M}} b_{3}<_{\bar{M}} b_{2}$, these half-edges are intertwined, and we know that the slicing of $\bar{M}$ by these half-edges creates a well-defined unicellular map $M$ of genus $g$ (Lemma2 2. Now, if we compare Figures 7 and 6 , we see that the result of the slicing is a triple of vertices $v_{1}, v_{2}, v_{3}$, such that each half-edge $b_{i}$ is the minimum in the vertex $v_{i}$ : indeed, the blocks $A_{1}, A_{2}, A_{3}, B_{2}, B_{3}$ are empty by construction, and the block $C_{3}=K$ is empty since $\tau$ is a trisection of type $\mathbf{I}$. Hence we have $\Phi\left(M, v_{1}, v_{2}, v_{3}\right)=(\bar{M}, \tau)$, so that the image of $\Phi$ exactly equals the set $\mathcal{D}_{g+1}^{I}(n)$.

\subsection{Trisections of type $\mathbf{I I}$.}

Of course, it would be nice to have a similar result for trisections of type II. Let $\bar{M}=(H, \alpha, \bar{\sigma})$ be a map of genus $g+1$ with a distinguished trisection $\tau$ of type II. We let $b_{1}, b_{2}, b_{3}$ and $K$ be as in Definition 3 and Figure 7, and we let $M$ be the result of the slicing of $\bar{M}$ at the three half-edges $b_{1}, b_{2}, b_{3}$. If we use the notations of Figure 6, with $a_{i}=b_{i}$, we see that we obtain three vertices, of diagrams $\Delta_{1}, \Delta_{2}, \Delta_{3}$, such that $A_{1}=A_{2}=B_{2}=A_{3}=B_{3}=\varnothing$. Hence, we know that $a_{1}=\min _{M}\left(v_{1}\right)$, that $a_{2}=\min _{M}\left(v_{2}\right)$, and that $a_{2}<\min _{M}\left(v_{3}\right)$. Observe that, contrarily to what happened in the previous section, the block $C_{3}=K$ is not empty, therefore $a_{3}$ is not the minimum inside its vertex.

Now, we claim that $\tau$ is still a trisection in the map $M$. Indeed, by construction, we know that $\tau$ belongs to $D_{3}$ (since, by definition of a trisection, it must be above $a_{3}$ in the map $\bar{M}$, and since $B_{3}$ is empty). Hence we still have $a_{3}<_{M} \tau$ in the map $M$. Moreover, we have clearly $\sigma(\tau)=a_{3}$ in $M$ (since 
$\tau$ is the rightmost point in the blocks $C_{3} \cup D_{3}$ ), and it follows that $\tau$ is a trisection in $M$.

We let $\Gamma(\bar{M}, \tau)=\left(M, v_{1}, v_{2}, \tau\right)$ be the 4-tuple consisting of the new map $M$, the two first vertices $v_{1}$ and $v_{2}$ obtained from the slicing, and the trisection $\tau$. It is clear that $\Gamma$ is injective: given $\left(\bar{M}, v_{1}, v_{2}, \tau\right)$, one can reconstruct the map $\bar{M}$ by letting $a_{1}=\min v_{1}, a_{2}=\min v_{2}$, and $a_{3}=\sigma(\tau)$, and by gluing back together the three half-edges $a_{1}, a_{2}, a_{3}$. Conversely, we define:

Definition 4. We let $\mathcal{V}_{g}(n)$ be the set of 4-tuples $\left(M, v_{1}, v_{2}, \tau\right)$, where $M$ is a unicellular map of genus $g$ with $n$ edges, and where $v_{1}, v_{2}$, and $\tau$ are respectively two vertices and a trisection of $M$ such that:

$$
\min _{M} v_{1}<_{M} \min _{M} v_{2}<_{M} \min _{M} V(\tau) .
$$

Given $\left(M, v_{1}, v_{2}, \tau\right) \in \mathcal{V}_{G}(n)$, we let $\bar{M}$ be the map obtained from the gluing of the three half-edges $\min v_{1}, \min v_{2}$, and $\sigma(\tau)$, and we let $\Psi\left(M, v_{1}, v_{2}, \tau\right):=(\bar{M}, \tau)$.

We can now state the following proposition, that completes Proposition 1 .

Proposition 2. The mapping $\Psi$ is a bijection between the set $\mathcal{V}_{g}(n)$ of unicellular maps of genus $g$ with $n$ edges a distinguished triple $\left(v_{1}, v_{2}, \tau\right)$ satisfying Equation 6 and the set $\mathcal{D}_{g+1}^{I I}(n)$ of unicellular maps of genus $g+1$ with $n$ edges and a distinguished trisection of type $\mathbf{I I}$.

Proof: In the discussion above, we have already given a mapping $\Gamma: \mathcal{D}_{g+1}^{I I}(n) \rightarrow \mathcal{V}_{g}(n)$, such that $\Psi \circ \Gamma$ is the identity on $\mathcal{D}_{g+1}^{I I}(n)$.

Conversely, let $\left(M, v_{1}, v_{2}, \tau\right) \in \mathcal{V}_{g}(n)$, and let $a_{1}=\min v_{1}, a_{2}=\min v_{2}$, and $a_{3}=\sigma(\tau)$. By definition, we know that $a_{2}<\min V(\tau)$, so that in the diagram representation of the three vertices $v_{1}, v_{2}, V(\tau)$ (Figure 6(a)) we know that the blocks $A_{1}, A_{2}, A_{3}, B_{2}, B_{3}$ are empty. Moreover, since $\tau$ is a trisection, $a_{3}$ is not the minimum inside its vertex, so the block $C_{3}$ is not empty. Hence, comparing Figures 6(c) and 7) and observing once again that the blocks $C_{3}$ and $K$ coincide, we see that after the gluing, $\tau$ is a trisection of type $\mathbf{I I}$ in the new map $\bar{M}$. Moreover, since the slicing and gluing operations are inverse one to the other, it is clear that $\Gamma(\bar{M}, \tau)=\left(M, v_{1}, v_{2}, \tau\right)$. Hence, $\Gamma \circ \Psi$ is the identity, and the proposition is proved.

\section{Iterating the bijection.}

Of course Proposition 1 looks nicer than its counterpart Proposition 2 in the first one, one only asks to distinguish three vertices in a map of lower genus, whereas in the second one, the marked triple must satisfy a nontrivial constraint (Equation 6). In this section we will work a little more in order to get rid of this problem. We start with two definitions (observe that for $k=3$ this is coherent with what precedes):

Definition 5. We let $\mathcal{U}_{g}^{k}(n)$ be the set of unicellular maps of genus $g$ with $n$ edges, and $k$ distinct distinguished vertices, undistinguishable one from the others.

Definition 6. We let $\mathcal{D}_{g}(n)=\mathcal{D}_{g}^{I}(n) \cup \mathcal{D}_{g}^{I I}(n)$ be the set of unicellular maps of genus $g$ with $n$ edges, and a distinguished trisection.

\subsection{Training examples: genera 1 and 2 .}

Observe that the set $\mathcal{V}_{0}(n)$ is empty, since there are no trisections in a plane tree. Hence, from Proposition 2 there are no trisections of type $\mathbf{I I}$ in a map of genus 1 (i.e. $\mathcal{D}_{1}^{I I}(n)=\varnothing$ ). Proposition 1 gives: 
Corollary 1. The set $D_{1}(n)$ of unicellular maps of genus 1 with $n$ edges and a distinguished trisection is in bijection with the set $U_{0}^{3}(n)$ of rooted plane trees with $n$ edges and three distinguished vertices.

We now consider the case of genus 2. Let $M$ be a unicellular map of genus 2 , and $\tau$ be a trisection of $M$. If $\tau$ is of type $\mathbf{I}$, we know that we can use the application $\Phi^{-1}$, and obtain a unicellular map of genus 1 , with three distinguised vertices.

Similarly, if $\tau$ is of type II, we can apply the mapping $\Psi^{-1}$, and we are left with a unicellular map $M^{\prime}$ of genus 1 , and a marked triple $\left(v_{1}, v_{2}, \tau\right)$, such that $\min _{M^{\prime}} v_{1}<_{M^{\prime}} \min _{M^{\prime}} v_{2}<_{M^{\prime}} \min _{M^{\prime}} V(\tau)$. From now on, we use the more compact notation: $v_{1}<_{M^{\prime}} v_{2}<_{M^{\prime}} V(\tau)$, i.e. we do not write the min's anymore. The map $\left(M^{\prime}, \tau\right)$ is a unicellular map of genus 1 with a distinguished trisection: therefore we can apply the mapping $\Phi^{-1}$ to $\left(M^{\prime}, \tau\right)$. We obtain a plane tree $M^{\prime \prime}$, with three distinguished vertices $v_{3}, v_{4}, v_{5}$ inherited from the slicing of $\tau$ in $M^{\prime}$; since those three vertices are undistinguishable, we can assume that $v_{3}<_{M^{\prime \prime}} v_{4}<_{M^{\prime \prime}} v_{5}$. Observe that in $M^{\prime \prime}$ we also have the two marked vertices $v_{1}$ and $v_{2}$ inherited from the slicing of $\tau$ in $M$. Moreover the fact that $v_{1}<_{M^{\prime}} v_{2}<_{M^{\prime}} V(\tau)$ in $M^{\prime}$ implies that $v_{1}<_{M^{\prime \prime}} v_{2}<_{M^{\prime \prime}} v_{3}$ in $M^{\prime \prime}$ : indeed, the gluing operation does not modify the part of cycle $\gamma$ appearing between the root and the smallest glued half-edge, so that appearing before $V(\tau)$ in $M^{\prime}$ is equivalent to appearing before $v_{3}$ in $M^{\prime \prime}$. Hence, we are left with a plane tree $M^{\prime \prime}$, with five distinguished vertices $v_{1}<_{M^{\prime \prime}} v_{2}<_{M^{\prime \prime}} v_{3}<_{M^{\prime \prime}} v_{4}<_{M^{\prime \prime}} v_{5}$. Conversely, given such a 5 -tuple of vertices, it is always possible to glue the three last ones together by the mapping $\Phi$ to obtain a triple $\left(v_{1}, v_{2}, \tau\right)$ satisfying Equation 6 . and then to apply the mapping $\Psi$ to retrieve a map of genus 2 with a marked trisection of type II. This gives:

Corollary 2. The set $\mathcal{D}_{2}^{I I}(n)$ is in bijection with the set $\mathcal{U}_{0}^{5}(n)$ of plane trees with five distinguished vertices.

The set $\mathcal{D}_{2}(n)$ of unicellular maps of genus 2 with one marked trisection is in bijection with the set $\mathcal{U}_{1}^{3}(n) \cup \mathcal{U}_{0}^{5}(n)$.

\subsection{The general case, and our main theorem.}

We let $p \geq 0$ and $q \geq 1$ be two integers, and $\left(M, v_{*}\right)=\left(M, v_{1}, \ldots, v_{2 q+1}\right)$ be an element of $\mathcal{U}_{p}^{2 q+1}(n)$. Up to renumbering the vertices, we can assume that $v_{1}<_{M} v_{2}<_{M} \ldots<_{M} v_{2 q+1}$.

Definition 7. We consider the following procedure:

i. Glue the three last vertices $v_{2 q-1}, v_{2 q}, v_{2 q+1}$ together, via the mapping $\Phi$, in order to obtain a new map $M_{1}$ of genus $p+1$ with a distinguished trisection $\tau$ of type $\mathbf{I}$.

ii. for $i$ from 1 to $q-1$ do:

Let $\left(v_{2 q-2 i-1}, v_{2 q-2 i}, \tau\right)$ be the triple consisting of the last two vertices which have not been used until now, and the trisection $\tau$. Apply the mapping $\Psi$ to that triple, in order to obtain a new map $M_{i+1}$ of genus $p+i+1$, with a distinguished trisection $\tau$ of type II.

end for.

We let $\Lambda\left(M, v_{*}\right):=\left(M_{q}, \tau\right)$ be the map with a distinguished trisection obtained at the end of this procedure. Observe that if $q=1$, the distinguished trisection is of type $\mathbf{I}$, and that it is of type II otherwise.

The following Theorem can easily be proved from Propositions 1 and 2 by adapting the arguments we used in the particular case of genus 2 : 
Theorem 1 (Our main result). The application $\Lambda$ defines a bijection:

$$
\Lambda: \biguplus_{p=0}^{g-1} \mathcal{U}_{p}^{2 g-2 p+1}(n) \longrightarrow \mathcal{D}_{g}(n) .
$$

In other words, all unicellular maps of genus $g$ with a distinguished trisection can be obtained in a canonical way by starting with a map of lower genus with an odd number of distinguished vertices, and then applying once the mapping $\Phi$, and a certain number of times the mapping $\Psi$.

Given a map with a marked trisection $(M, \tau)$, the converse application consists in slicing recursively the trisection $\tau$ while it is of type $\mathbf{I I}$, then slicing once the obtained trisection of type $\mathbf{I}$, and remembering all the vertices resulting from the successive slicings.

Finally, our new identity (Equation (4)) follows from the theorem and the Trisection lemma (Lemma3). Further developments: - It is known that labelled unicellular maps are in bijection with general maps of the same genus (this is the Marcus-Schaeffer bijection). Hence our bijection also leads to a full description of maps of positive genus in terms of plane labelled trees with distinguished vertices.

- It is straightforward to obtain a formula analogous to (4) for the numbers $\beta_{g}(k, l)$ of bipartite unicellular maps with $k$ white and $l$ black vertices (just be careful to glue only vertices of the same color).

- It is possible to iterate the mapping $\Lambda$ in order to obtain only plane trees at the end. This leads to the following formula, which interestingly reminds of Equation 1 ;

$$
\epsilon_{g}(n)=\left(\sum_{0=g_{0}<g_{1}<\ldots<g_{r}=g} \prod_{i=1}^{r} \frac{1}{2 g_{i}}\left(\begin{array}{c}
n+1-2 g_{i-i} \\
2\left(g_{i}-g_{i-1}\right)+1
\end{array}\right)\right) \operatorname{Cat}(n) .
$$

\section{References}

[GN05] I. P. Goulden and A. Nica. A direct bijection for the Harer-Zagier formula. J. Combin. Theory Ser. A, 111(2):224-238, 2005.

[GS98] Alain Goupil and Gilles Schaeffer. Factoring $n$-cycles and counting maps of given genus. European J. Combin., 19(7):819-834, 1998.

[HZ86] J. Harer and D. Zagier. The Euler characteristic of the moduli space of curves. Invent. Math., 85(3):457-485, 1986

[Jac87] D. M. Jackson. Counting cycles in permutations by group characters, with an application to a topological problem. Trans. Amer. Math. Soc., 299(2):785-801, 1987.

[Las01] Bodo Lass. Démonstration combinatoire de la formule de Harer-Zagier. C. R. Acad. Sci. Paris Sér. I Math., 333(3):155-160, 2001.

[MT01] Bojan Mohar and Carsten Thomassen. Graphs on surfaces. Johns Hopkins Studies in the Mathematical Sciences. Johns Hopkins University Press, Baltimore, MD, 2001.

[SV08] Gilles Schaeffer and Ekaterina Vassilieva. A bijective proof of Jackson's formula for the number of factorizations of a cycle. J. Combin. Theory Ser. A, 115(6):903-924, 2008.

[WL72] T. R. S. Walsh and A. B. Lehman. Counting rooted maps by genus. I. J. Combinatorial Theory Ser. B, 13:192-218, 1972. 